\title{
Terahertz-driven, sub-keV electron gun
}

\author{
W. Ronny Huang ${ }^{1,2}$, Arya Fallahi' ${ }^{2}$ Xiaojun Wu ${ }^{2}$, Emilio A. Nanni ${ }^{1}$, Huseyin Cankaya ${ }^{2}$, Anne-Laure \\ Calendron' ${ }^{2}$, Dongfang Zhang ${ }^{2}$, Koustuban Ravi ${ }^{1,2}$, Kyung-Han Hong ${ }^{1}$, Franz X. Kärtner ${ }^{1,2,3}$ \\ ${ }^{1}$ Department of Electrical Engineering and Computer Science and Research Laboratory of Electronics, Massachusetts Institute of Technology, \\ Cambridge, MA 02139, USA \\ ${ }^{2}$ Center for Free-Electron Laser Science, Deutsches Elektronen-Synchrotron DESY, Notkestraße 85, 22607 Hamburg, Germany \\ ${ }^{3}$ Physics Department and The Hamburg Center for Ultrafast Imaging, University of Hamburg, Luruper Chaussee 149, 22761 Hamburg, \\ Germany \\ franz.kaertner@desy.de
}

\begin{abstract}
Strong-field, single-cycle $\mathrm{THz}$ fields accelerate electrons with peak energies of up to $0.75 \mathrm{keV}$ in a millimeter-sized $\mathrm{THz}$ gun with bunch charge of $45 \mathrm{fC}$. Energy spreads as low as $5.8 \%$ were also achieved.

OCIS codes: (320.7110) Ultrafast nonlinear optics; (320.7080) Ultrafast devices; (140.3070) Infrared, far-infrared lasers
\end{abstract}

\section{Introduction}

Ultrafast electron guns are an enabling technology for femtosecond electron diffraction and compact free electron lasers. Guns accelerate electrons either by DC electrostatic fields or by synchronized RF fields. Current hurdles facing the construction of guns include the bulky, expensive high voltage feedthroughs or high power klystrons needed to power DC or RF guns, respectively, as well as the synchronization challenges faced by RF guns. Further, both DC and RF guns show limitations in the accelerating field of about $10 \mathrm{MV} / \mathrm{m}$ and $200 \mathrm{MV} / \mathrm{m}$, respectively, due to breakdown mechanisms in common accelerator materials [1]. It is well known in accelerator physics that electron beam size and bunch duration directly achieved from the gun is inversely proportional to the accelerating electric field. The need for more economical devices, coupled with the desire for higher field strength, has propelled the development of compact NIR- or THz-driven linear accelerator (linacs) structures with higher field breakdown thresholds [2,3] and intrinsic synchronization. The enormous potential advantages of light-driven linacs, however, has not extended to guns [4], which are essential to determining beam quality. Here we implement a first-version of THz-driven electron gun based on a parallel plate waveguide (PPWG) structure with subwavelength field confinement. We report on the demonstration of $\mathrm{keV}$-scale acceleration of a $45 \mathrm{fC}$ electron bunch using a singlecycle THz pulse generated by a modest mJ-scale NIR laser. Energy spreads as low as $5.8 \%$ were achieved.

\section{Experimental setup}
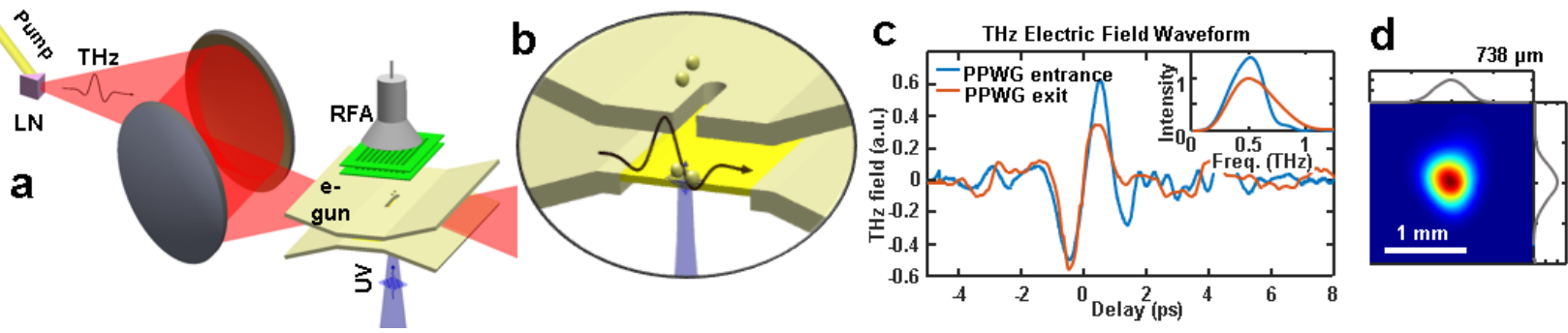

Figure 1: Schematic of setup (a) zoomed out and (b) cross-section zoomed in. (c) THz electric waveform and spectrum (inset) at the entrance and exit of the PPWG gun structure. (d) THz intensity profile at free-space focus.

The THz Gun structure, schematic in Fig. 1(a)-(b), consists of a copper PPWG, an angled coupling taper, a thin-film copper photocathode, and an exit anode. A THz beam, polarized perpendicularly to the PPWG walls, is coupled into it by the assistance of the angled taper. Once inside the waveguide, the $\mathrm{THz}$ electric field accelerates an electron bunch, produced via photoelectric emission by UV back-illumination of the photocathode. Accelerated electrons travel across the vertical extent of the waveguide and exit through a narrow slit on the anode. Afterward, they are detected and spectrally characterized by a retarding field analyzer (RFA). The THz pulses are generated by optical rectification in lithium niobate using the tilted pulse front technique. The energy impinging the gun was $33 \mu \mathrm{J}$ after relaying off parabolic mirrors. Electro-optic sampling (EOS) at the gun "entrance" (at the focus of the parabolic mirror with the gun removed) and at the gun "exit" (at the focus of a second relay after the THz travels through the waveguide-not shown in schematic) reveals single-cycle temporal waveforms of about 1.2 ps FWHM (Fig. 1(c)). A horizontal beam waist of $0.74 \mathrm{~mm}$ at the gun (Fig. 1(d)). Taking into account the energy, waveform, and beam waist, the focused $\mathrm{THz}$ has a calculated peak field in the range of $100 \mathrm{MV} / \mathrm{m}$ in free space. Assuming the horizontal 
beam profile remains unaltered while the vertical profile is distributed uniformly across the $75 \mu \mathrm{m}$ spacing, and taking into account the coupling efficiency, the confined THz pulse inside the PPWG has a peak field in the range of $300 \mathrm{MV} / \mathrm{m}$.

\section{Results}

We record the kinetic energy spectra as a function of delay between the THz wave and UV photoemitter, producing a streaking spectrogram in Fig. 2(a). When the emission occurs before the terahertz pulse $(<-2 \mathrm{ps})$, we observe a large spread of kinetic energies, resulting from UV emission combined with THz field emission, a phenomenon observed and investigated in [5]. Conversely, when the emission occurs during the terahertz pulse (-2 to 2 ps), we observe a narrow energy spread with energy gain governed by the $\mathrm{THz}$ phase at which emission occurs. Between 0.3-0.8 ps, we observe suppression of photocurrent resulting from the fact that emission occurs at the highest downward force of the THz field. Two delay positions were selected to analyze the THz energy dependence, plotted in Fig. 2(b)-(c). The first delay, $\tau_{1}$, produced the highest electron energies while the second delay, $\tau_{2}$, produced the most monochromatic spectra. Each spectrum exhibits a unimodal distribution with a peak increasing monotonically with $\mathrm{THz}$ field. The spectral shape is asymmetric with a peak yield toward higher energies, followed by a sharp cutoff, and a pedestal toward lower energies. The high yield near the cutoff indicate that most of the electrons are emitted at the optimal $\mathrm{THz}$ phase and concurrently experience the same acceleration. The pedestal can be attributed to the electrons which are emitted away from the optimal phase, resulting in relatively less energy gain. At $\tau_{1}$, we achieve energy gain of $0.75 \mathrm{keV}$ for maximal THz energy. The bunch charge was measured to be $45 \mathrm{fC}$ at this setting.
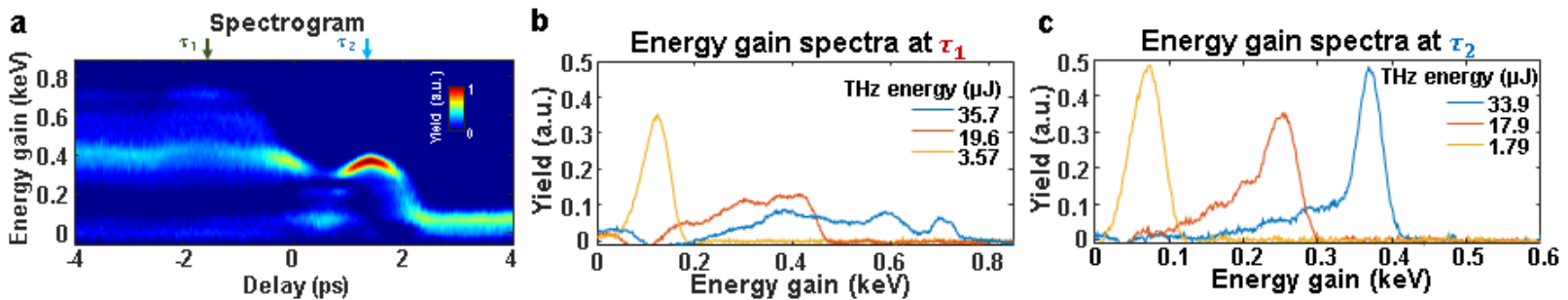

Figure 2: (c) Spectrogram showing spectra as function of delay between UV and THz, at max THz energy. (b)-(c) Accelerated electron spectra at delay (a) $\tau_{1}$ and (b) $\tau_{2}$.

By plotting the spectra as a function of THz energy on a 2D map in Fig. 3(a)-(b), it is observed that the energy gain increases monotonically with the THz energy. We also observe a monotonically decreasing percent RMS energy spread with accelerating energy. The lowest energy spreads achieved at $\tau_{1}$ and $\tau_{2}$ were $26 \%$ and $5.8 \%$ for $\mathrm{THz}$ energies of $35.7 \mu \mathrm{J}$ and $33.9 \mu \mathrm{J}$, respectively.

\section{a}

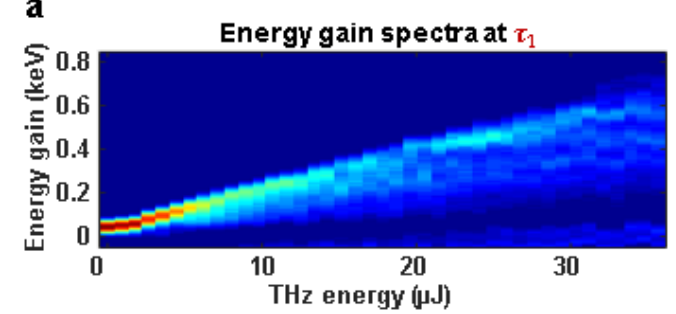

Figure 3: Accelerated electron spectra as a function of THz energy at delay (a) $\tau_{1}$ and (b) $\tau_{2}$.

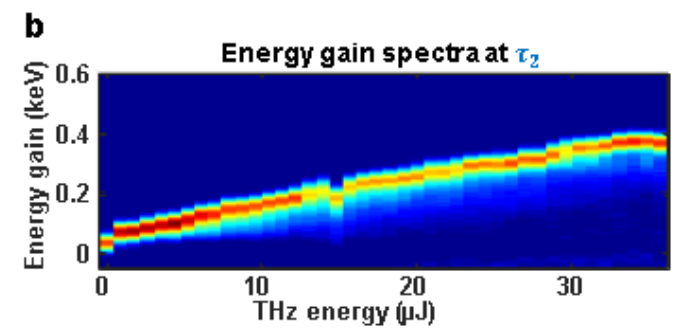

b

\section{Conclusion}

We have built a first electron gun driven by strong THz fields, with close to $1 \mathrm{keV}$-scale acceleration. The gun was operated over the entire 4 days of period without noticeable degradation.

\section{References}

[1] B. Spataro et al., "Technological issues and high gradient test results on X-band molybdenum accelerating structures,” Nucl. Instruments Methods Phys. Res. A 657, 114-121 (2011).

[2] K. J. Leedle, R. Fabian Pease, R. L. Byer, and J. S. Harris, "Laser acceleration and deflection of 96.3 keV electrons with a silicon dielectric structure," Optica 2, 158 (2015).

[3] E.A. Nanni et al., "Terahertz-driven linear electron acceleration,” Nature Communications 6, 8486 (2015).

[4] W. R. Huang et al., “Toward a terahertz-driven electron gun,” Scientific Reports 5, 14899 (2015).

[5] G. Herink, L. Wimmer, and C. Ropers, "Field emission at terahertz frequencies: AC-tunneling and ultrafast carrier dynamics,” New J. Phys. 16, 123005 (2014). 